\title{
Collective excitation of a Bose-Einstein condensate by modulation of the atomic scattering length
}

\author{
S. E. Pollack, D. Dries, and R. G. Hulet \\ Department of Physics and Astronomy and Rice Quantum Institute, Rice University, Houston, Texas 77005, USA \\ K. M. F. Magalhães, E. A. L. Henn, E. R. F. Ramos, M. A. Caracanhas, and V. S. Bagnato \\ Instituto de Física de São Carlos, Universidade de São Paulo, Caixa Postal 369, 13560-970 São Carlos-SP, Brazil
}

(Received 12 January 2010; published 24 May 2010)

\begin{abstract}
We excite the lowest-lying quadrupole mode of a Bose-Einstein condensate by modulating the atomic scattering length via a Feshbach resonance. Excitation occurs at various modulation frequencies, and resonances located at the natural quadrupole frequency of the condensate and at the first harmonic are observed. We also investigate the amplitude of the excited mode as a function of modulation depth. Numerical simulations based on a variational calculation agree with our experimental results and provide insight into the observed behavior.
\end{abstract}

DOI: 10.1103/PhysRevA.81.053627

PACS number(s): 03.75.Kk, 03.75.Nt, 67.85.De

Collective excitation of a Bose-Einstein condensate (BEC) is an essential diagnostic tool for investigating properties of the ultracold quantum state. Fundamental information about condensate dynamics can be determined from observations of collective modes [1-3], including the effects of temperature [4-6], and the dimensionality of the system [7]. In addition, the interplay between these modes and external agents, such as random potentials [8,9], lattices [10,11], as well as other atoms [12-16], can be investigated. Examining the excitation spectrum of the BEC allows for a detailed comparison with theoretical models [17-20] and related quantum systems such as superfluid helium [21,22] and superconductors [23,24].

Generically, a collective excitation is generated by the modification of the trapping potential of the condensate [25]. One convenient method is to apply a sudden magnetic field gradient, thereby shifting the center of the trap and exciting a dipole oscillation about the trap center. One may also suddenly change the curvature of the trap to excite quadrupole modes. The lowest-lying $m=0$ quadrupole mode is characterized by out-of-phase axial and radial oscillations.

If the condensate is not the only occupant of the trap (i.e., there exists a thermal component or another species of atoms) then the other atoms may also be excited through these processes. The evolution of a collective excitation can therefore be complicated because the multiple components may affect damping or induce frequency shifts of the oscillation [12-16]. Therefore, modulating the trap, although an extremely useful tool for an isolated condensate, can be cumbersome when the system to be studied is multispecied. An alternative approach is to excite the condensate alone, leaving the other occupants of the trap untouched.

In this work, we demonstrate the excitation of the lowestlying quadrupole mode in a $\mathrm{BEC}$ of ${ }^{7} \mathrm{Li}$ by modulating the atomic scattering length via a magnetic Feshbach resonance. In contrast to abruptly changing the scattering length [26], sinusoidal modulation enables the controlled excitation of a single mode at a specific frequency. In addition, by using this method, a coexisting thermal component will be minimally excited by the mean-field coupling to the normal gas [27,28]. In the case of a multispecies experiment, resonant modulation of the scattering length of one species will not necessarily excite the others, depending on the details of other Feshbach resonances present in the system [29]. Therefore, this technique may be useful for investigating nonzero temperature effects and as a powerful diagnostic tool for multispecies ultracold atomic experiments.

A trapped BEC at zero temperature may be described by the three-dimensional cylindrically symmetric Gross-Pitaevskii equation [30]

$$
i \hbar \frac{\partial}{\partial t} \psi=-\frac{\hbar^{2}}{2 m} \nabla^{2} \psi+V \psi+\frac{4 \pi \hbar^{2} a}{m}|\psi|^{2} \psi,
$$

where $m$ is the atomic mass, the trapping potential is $V=$ $(1 / 2) m\left(\omega_{r}^{2} r^{2}+\omega_{z}^{2} z^{2}\right)$ with $\omega_{z}\left(\omega_{r}\right)$ the axial (radial) trapping frequency, $a$ is the $s$-wave scattering length, and the density is given by $n=|\psi|^{2}$. It is convenient to introduce the anisotropy parameter $\lambda=\omega_{z} / \omega_{r}$. We use a variational approach to solve this equation and determine the frequencies of the lowestlying modes. Using a Gaussian ansatz and minimizing the corresponding energy functional, we derive the following coupled differential equations for the dimensionless axial and radial half-widths $u_{z}$ and $u_{r}$ of the condensate [31]:

$$
\begin{gathered}
\ddot{u}_{r}+u_{r}=\frac{1}{u_{r}^{3}}+\frac{P}{u_{r}^{3} u_{z}}, \\
\ddot{u}_{z}+\lambda^{2} u_{z}=\frac{1}{u_{z}^{3}}+\frac{P}{u_{r}^{2} u_{z}^{2}},
\end{gathered}
$$

where the interaction parameter $P=\sqrt{2 / \pi}\left(N a / l_{r}\right), l_{r}=$ $\sqrt{\hbar / m \omega_{r}}$ is the radial harmonic oscillator size, and $N$ is the number of condensed atoms. We solve Eq. (2) in the case of harmonic motion of the Gaussian sizes about their equilibrium values. The frequency of the lowest-lying quadrupole oscillation is [31]

$$
\begin{aligned}
\omega_{Q}= & \omega_{r} \sqrt{2}\left[\left(1+\lambda^{2}-P_{2,3}\right)\right. \\
& \left.-\sqrt{\left(1-\lambda^{2}+P_{2,3}\right)^{2}+8 P_{3,2}^{2}}\right]^{1 / 2},
\end{aligned}
$$

where $P_{i, j}=P /\left(4 u_{0 r}^{i} u_{0 z}^{j}\right)$ and $u_{0 z}$ and $u_{0 r}$ are the equilibrium axial and radial sizes, respectively. The frequency of the $m=0$ breathing mode is the sum of the two terms in Eq. (3) rather than the difference and is a factor of about 60 higher in 
frequency for our experimental parameters. For the case of a highly elongated trap $(\lambda \ll 1)$, in the Thomas-Fermi regime $(P \gg 1)$, we find the well-known relation $\omega_{Q}=\omega_{z} \sqrt{5 / 2}$ $[18,32]$, and in the noninteracting limit $(P \rightarrow 0)$, we find $\omega_{Q} \rightarrow 2 \omega_{z}$, as expected.

We may also use Eq. (2) to determine the time evolution of the size of the BEC [33]. In particular, we are interested in the dynamics associated with the modulation of $a$ using a Feshbach resonance, which has been proposed previously [34,35]. In a magnetic field $B$, the scattering length near a Feshbach resonance may be described by

$$
a(B)=a_{\mathrm{BG}}\left(1-\frac{\Delta}{B-B_{\infty}}\right),
$$

where $a_{\mathrm{BG}}$ is the background scattering length, $\Delta$ is the resonance width, and $B_{\infty}$ is the resonance location. We consider a time-dependent magnetic field of the form

$$
B(t)=B_{\mathrm{av}}+\delta B \cos (\Omega t),
$$

where $\Omega$ is the modulation frequency. Using this form for $B$, the result of expanding Eq. (4) to first order in the small quantity $\delta B \ll\left|B_{\mathrm{av}}-B_{\infty}\right|$ is

$$
a(t) \simeq a_{\mathrm{av}}+\delta a \cos (\Omega t)
$$

where

$$
a_{\mathrm{av}}=a\left(B_{\mathrm{av}}\right) \quad \text { and } \quad \delta a=\frac{a_{\mathrm{BG}} \Delta \delta B}{\left(B_{\mathrm{av}}-B_{\infty}\right)^{2}} .
$$

This expression for $a$ is substituted into Eq. (2), and a fourth-order Runge-Kutta method is used to solve the system of equations numerically. The results may be directly compared with those from experiment.

Our methods for creating an ultracold gas of ${ }^{7} \mathrm{Li}$ have been described previously [36,37]. We confine atoms in the $|1,1\rangle$ hyperfine state of ${ }^{7} \mathrm{Li}$ in an optical trap and use a set of Helmholtz coils to provide an axially oriented bias field. We determine the radial trapping frequency by parametric heating to be $\omega_{r}=(2 \pi) 235(10) \mathrm{Hz}$, and the axial trapping frequency by dipole oscillation to be $\omega_{z}=(2 \pi) 4.85(1)$ Hz. After evaporation from the optical trap at $717 \mathrm{G}$, where $a \sim 200 a_{0}$, we have $N \sim 3 \times 10^{5}$ atoms with a condensate fraction $>90 \%$. We then ramp the bias field in $4 \mathrm{~s}$ from the initial value to $B_{\mathrm{av}}=565 \mathrm{G}$ (where $a_{\mathrm{av}} \sim 3 a_{0}$ ). For these experimental values, the dimensionality parameter is $\lambda P \approx 0.3$, close to the transition into the quasi-one-dimensional regime (determined by $\lambda P \ll 1)$ [38]. At this point we oscillate the magnetic field with a modulation depth of $\delta B=14 \mathrm{G}$, corresponding to $\delta a \sim 2 a_{0}$. We use in situ polarization phase-contrast imaging to obtain the density distribution of the condensate [39], to which we fit a Gaussian characterized by $1 / e$ axial and radial half-widths.

In Fig. 1, we show pictures taken with $\Omega=(2 \pi) 10 \mathrm{~Hz}$. A quadrupole oscillation of the cloud is readily observable. The large oscillation amplitudes considered here extend over approximately $1 \mathrm{~mm}$ of the optical trap. A harmonic approximation of the trapping potential about the trap center is less than $10 \%$ in error over this range. The size of the cloud in Fig. 1 as a function of time is modeled well by the variational calculation, consistent with negligible anharmonic

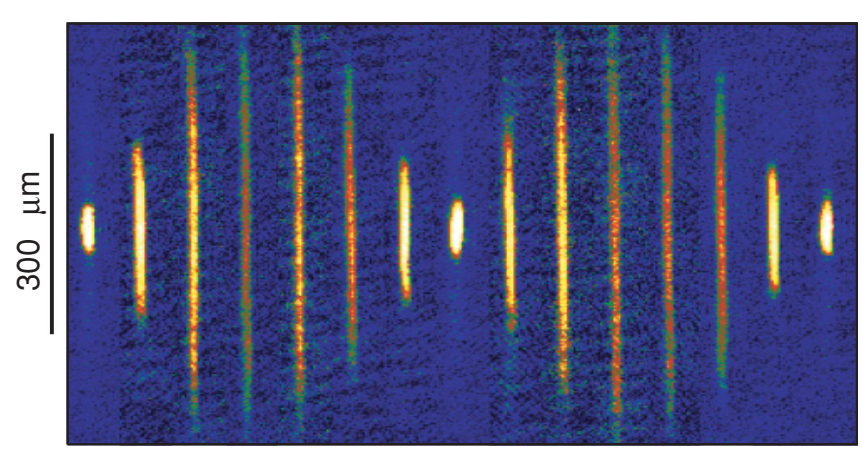

FIG. 1. (Color online) Quadrupole oscillation excited by the modulation of $a$. A series of in situ polarization phase-contrast images of condensates taken during excitation at $\Omega=(2 \pi) 10 \mathrm{~Hz}$, separated in time by $15 \mathrm{~ms}$. The change in peak density is nearly an order of magnitude from the most compressed to the most extended condensate. The horizontal scale of these images has been stretched by a factor of 2 for clarity. There is negligible excitation of the dipole mode.

contributions. Furthermore, we observe no damping of the quadrupole mode over many oscillation periods, consistent with a negligible thermal fraction.

Results from the variational calculation show that, during the excitation, if $\Omega<\omega_{Q}$ then the axial and radial sizes of the cloud follow the change in $a$ : growing as $a$ increases, and shrinking as $a$ decreases. This in-phase behavior of both the axial and radial sizes of the cloud is expected for adiabatic changes in $a$ and, therefore, should not be confused with the high-lying $m=0$ breathing mode for low frequencies. However, when $\Omega>\omega_{Q}$, the radial size follows nearly in phase, while the axial size lags behind the radial size by half a period-an out-of-phase oscillation. In both cases when the excitation is stopped, the cloud undergoes free quadrupole oscillations with the axial and radial sizes $\pi$ out of phase. Characteristic data for these two regimes are shown in Fig. 2 and reasonably agree with the variational results. We fit the time evolution of the size of the cloud after excitation and determine the free quadrupolar oscillation frequency to be $\omega_{Q}=$ $(2 \pi) 8.2(1) \mathrm{Hz}$, in good agreement with the predicted value of $8.17 \mathrm{~Hz}$ from Eq. (3), where we have used $\lambda=0.021$ and $P \approx 15$. Similar agreement between measured and predicted quadrupole frequencies in the dimensional crossover regime have been previously observed [7]. The amplitude of this free oscillation is dependent on the duration of excitation as well as the phase of the driving force at the time when the excitation is stopped. Therefore, care must be taken when comparing data with theoretical predictions of the amplitude during the free oscillation.

A less parameter-dependent measure is to observe the amplitude of the oscillation during excitation. As can be discerned from Fig. 2, during excitation the size of the condensate oscillates at both the drive frequency and the natural quadrupole frequency. Beating between these frequencies modulates the instantaneous deviation from the unperturbed size. An ideal method for determining the energy in the driven mode and the excited quadrupole mode separately is to use Fourier analysis [40]. This method can be experimentally difficult, however, given the required number of coherent 


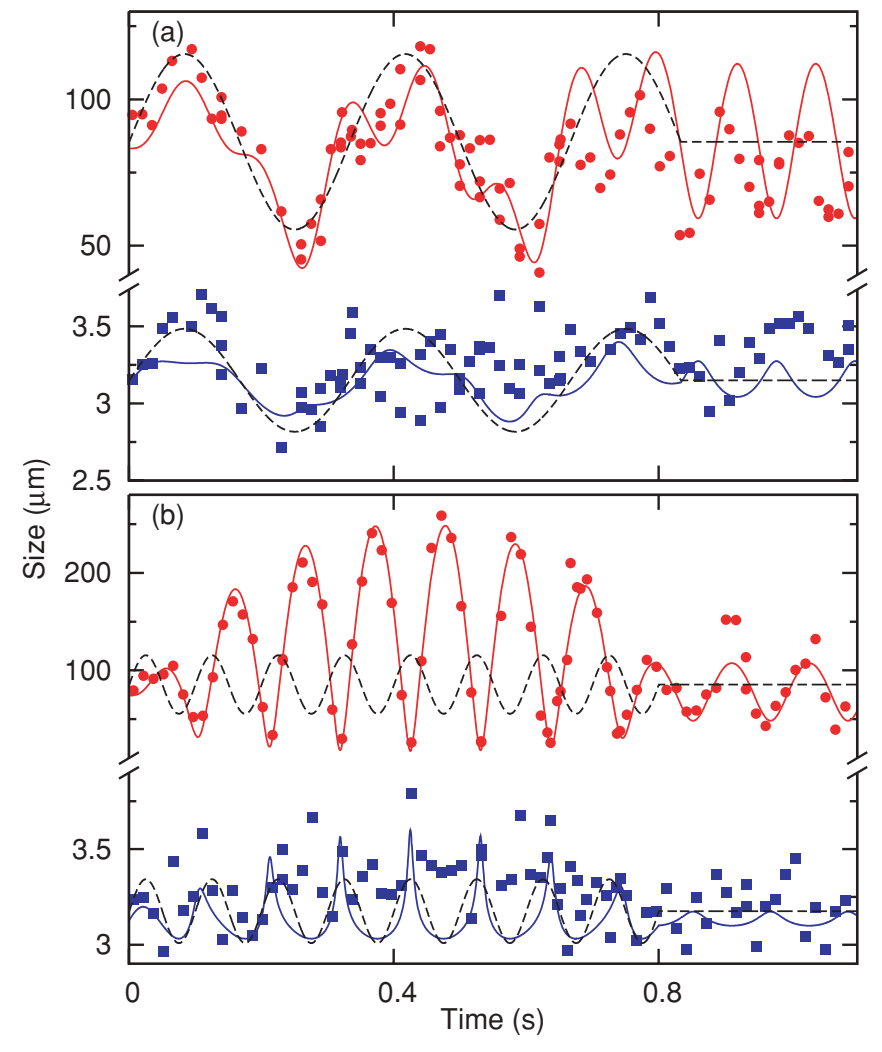

FIG. 2. (Color online) Axial [(red) circles] and radial [(blue) squares] $1 / e$ sizes of a condensate during and after excitation with $a_{\mathrm{av}} \sim 3 a_{0}$ and $\delta a \sim 2 a_{0}$, where (a) $\Omega=(2 \pi) 3 \mathrm{~Hz}$ or (b) $\Omega=$ $(2 \pi) 10 \mathrm{~Hz}$. The natural oscillation frequency is $\omega_{Q}=(2 \pi) 8.2(1) \mathrm{Hz}$. Note that the $1.8-\mathrm{Hz}$ beat note is the cause of the diminished amplitude in (b) after the modulation drive is turned off. The solid lines are results of the variational calculation using the same parameters as in the experiment and contain no adjustable parameters. The dashed lines are representative of the driving excitation. The resolution of the optical imaging system is $\sim 3 \mu \mathrm{m}$, which limits accurate determination of the radial sizes. The frequency of the breathing mode is on the order of $470 \mathrm{~Hz}$ and therefore no effects of this mode are present.

oscillations needed to accurately resolve the sinusoidal peak in the Fourier spectrum. Instead, we assume that the system can be described by the linear combination of sinusoids at the known frequencies $\Omega$ and $\omega_{Q}$. After driving an excitation for $0.5 \mathrm{~s}$, we fit the observed condensate axial size to the following expression during an additional $0.5 \mathrm{~s}$ of excitation:

$$
u(t)=u_{0}+u_{\Omega} \sin (\Omega t+\Phi)+u_{Q} \sin \left(\omega_{Q} t+\phi\right),
$$

where $u_{0}$ is the equilibrium $1 / e$ size, $u_{\Omega}$ and $u_{Q}$ are the amplitudes of the drive and quadrupole modes, respectively, and $\Phi$ and $\phi$ are the respective phases. The fractional amplitudes $u_{\Omega} / u_{0}$ and $u_{Q} / u_{0}$ are shown as functions of $\Omega$ in Fig. 3. As expected, there is a resonant enhancement in both the quadrupole and the drive modes when $\Omega=\omega_{Q}$. In addition, we see a parametric enhancement when $\Omega \approx 2 \omega_{Q}$. The dip at exactly $2 \omega_{Q}$ is due to destructive interference between the drive mode and the resonantly excited mode. This interference structure is observed in both the data and the simulation.

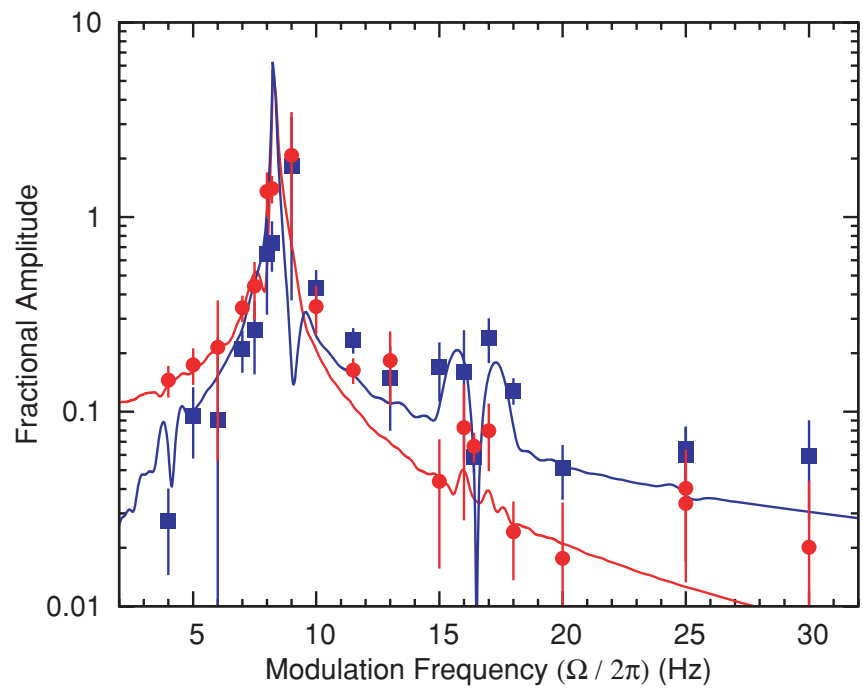

FIG. 3. (Color online) Fractional amplitude of the drive [(red) circles] and quadrupole [(blue) squares] modes during excitation at frequency $\Omega$ with a modulation depth $\delta a \sim 1 a_{0}$, where $a_{\mathrm{av}} \sim 3 a_{0}$. The solid lines are results of the variational calculation with no adjustable parameters. The oscillation is notably asymmetric for fractional amplitudes of order 1 and larger, as shown in Fig. 2(b).

Larger drive amplitudes push the oscillations into the nonlinear regime where the amplitude of oscillation is no longer linearly dependent on the modulation depth. The first noticeable effect is the nonsinusoidal behavior of the oscillation seen in Fig. 2(b). In Fig. 4 we show the result of drivingthe system near resonance with a fractional modulation depth $\delta a / a_{\mathrm{av}}=1$. As the amplitude of the driven oscillation grows, it eventually becomes comparable with the original condensate size. At this point, the size of the condensate cannot become smaller; therefore, the oscillation becomes increasingly asymmetric. In this manner, the rate of energy transfer into the oscillation will decrease. At even larger amplitudes, we observe that the condensate appears to fragment [35].

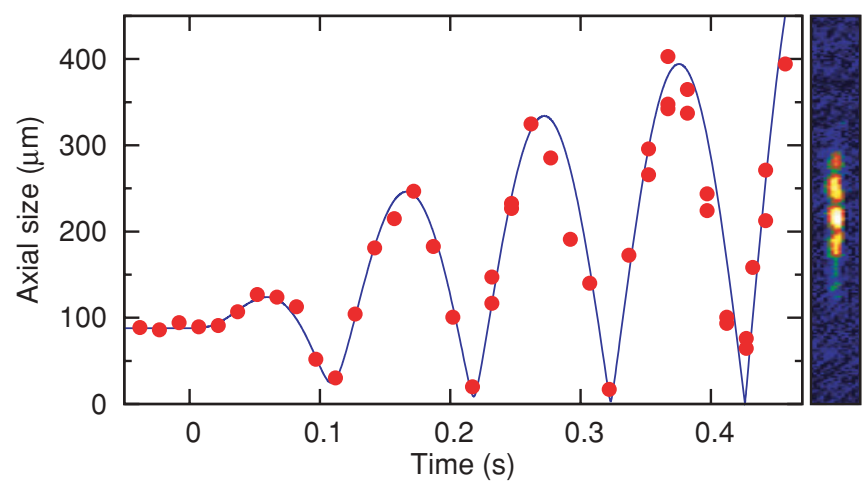

FIG. 4. (Color online) Quadrupole oscillation driven by a largeamplitude excitation, $\delta a \sim 3 a_{0}$ where $a_{\text {av }} \sim 3 a_{0}$, near resonance $\Omega=(2 \pi) 9 \mathrm{~Hz}$. The nonsinusoidal behavior leads to fragmentation of the condensate during the compression stages when the axial size becomes smaller than the axial harmonic oscillator size of $\sim 17 \mu \mathrm{m}$. The image at right was taken during the compression stage at $t=$ $0.52 \mathrm{~s}$ and shows fragmentation of the condensate; the field of view is $25 \times 380 \mu \mathrm{m}^{2}$. 


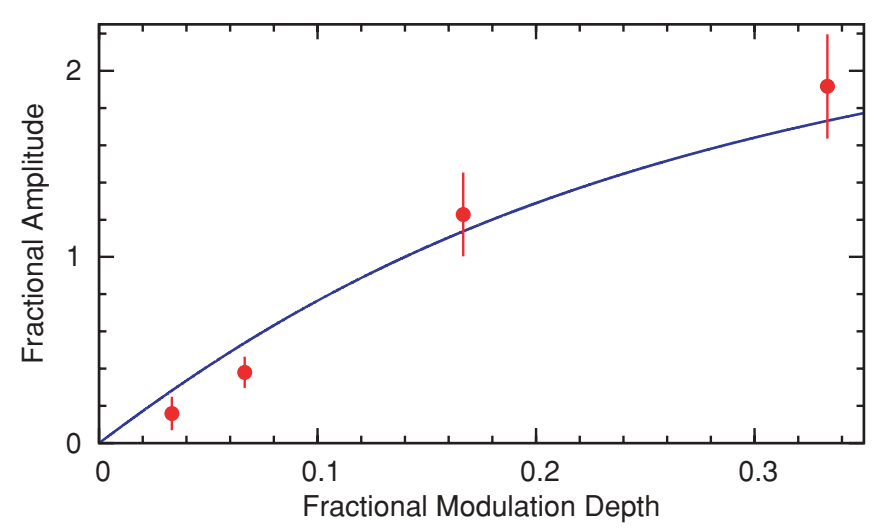

FIG. 5. (Color online) Fractional amplitude of oscillation, when $\Omega \approx \omega_{Q}$, as a function of the fractional modulation depth $\delta a / a_{\mathrm{av}}$ after $1 \mathrm{~s}$ of excitation. The solid curve is the result of the variational calculation.

Similar-looking results have been observed when modulating the radial confinement of a cigar-shaped BEC, where Faraday waves may be excited [41]. By fitting the excitation spectrum (Fig. 3) to a skew Lorentzian (where the peak excitation frequency, width, amplitude, and skewness are fit parameters), we determine the maximum fractional amplitude of oscillation of the quadrupole mode after $1 \mathrm{~s}$ of excitation. Our experimental results for the amplitude as a function of modulation depth are presented in Fig. 5 along with results from the variational calculation, which show good agreement with no adjustable parameters. We note that, for the smallest of modulation depths investigated, we only observed oscillation of the condensate when the drive frequency was near resonance. In addition, we found a roughly linear scaling of the instantaneous amplitude of the quadrupole oscillation with the duration of the excitation in this regime. Furthermore, Fig. 5 conveys to us that the data in Fig. 2, which were driven at a fractional modulation depth of $\sim 0.7$, were not in the linear regime, and the data shown in Fig. 3, driven at a fractional modulation depth of $\sim 0.3$, had a response that departed from linearity by about $30 \%$.

Large-amplitude oscillations are accompanied by a frequency shift of the quadrupole mode [42]. For our geometry, this shift can be approximated by $\delta \omega / \omega_{Q} \approx 0.1 A_{z}^{2}$ for small $A_{z}$, where $A_{z} \equiv u_{Q} / u_{0}$ is the fractional amplitude of the axial size [40]. The data shown in Fig. 3 have $A_{z} \sim 2$ on resonance, and therefore in this case the above approximation for the frequency shift is not valid. Our simulated results show a frequency shift of $\sim 8 \%$ for $A_{z}=2$ with a shallow amplitude dependence at larger $A_{z}$. Whereas a shift of $10 \%$ was observed in the oscillation of Fig. 4, we were not able to resolve frequency shifts by fitting Lorentzians to the excitation spectra for our data at low $A_{z}$ shown in Fig. 5. There is an additional frequency shift due to nonzero temperature [4-7] which is estimated to be negligible given our low temperatures and interaction strength [43]. Even though we can neglect temperature effects in the measurements presented here, our method of excitation of the quadrupole mode may be used to study these effects in further detail in regimes of stronger interactions. In addition, going to large values of $a$ will facilitate investigations of beyond mean-field effects on the collective modes of a Bose gas [44], complementary to those observed in a Fermi gas at the BCS-BEC crossover [45-47].

In this work, we have experimentally demonstrated the excitation of the collective low-lying quadrupole mode of a dilute Bose gas by modulating the atomic scattering length. Our observations are supported by variational calculations of the time-dependent Gross-Pitaevskii equation assuming a Gaussian trial wave function. Using this formalism we find good agreement with our experimental results. Temporal modulation of the scattering length, as afforded by Feshbach resonances, provides an additional tool for exciting collective modes of an ultracold atomic gas. This method is quite attractive in circumstances where excitation of the condensate by other means, such as trap deformation, is unavailable. In addition, this method can be used for condensates in the presence of thermal atoms where principal excitation of the condensate alone is desired, as well as in multicomponent gases where excitation of only one species can be accomplished.

\section{ACKNOWLEDGMENTS}

We thank V. I. Yukalov for stimulating discussions related to this work. The work at Rice was funded by the NSF, the ONR, the Keck Foundation, and the Welch Foundation (Grant No. C-1133). The work at São Carlos was funded by Fundaçao de Amparo a Pesquisa do Estado de Sao Paulo-Programa de Centros de Pesquisa e Inovaçao (FAPESP-CEPID), Centro Nacional de Pesquisa e Fundaçao de Amparo a Pesquisa do Estado de Sao Paulo-Programa de Insittutos Nacionais (CNPq/FAPESP-INCT), and Centro de Aperfeiçoamento de Ensino Superior (CAPES).
[1] D. S. Jin, J. R. Ensher, M. R. Matthews, C. E. Wieman, and E. A. Cornell, Phys. Rev. Lett. 77, 420 (1996).

[2] M.-O. Mewes, M. R. Andrews, N. J. van Druten, D. M. Kurn, D. S. Durfee, C. G. Townsend, and W. Ketterle, Phys. Rev. Lett. 77, 988 (1996).

[3] C. Fort, M. Prevedelli, F. Minardi, F. S. Cataliotti, L. Ricci, G. M. Tino, and M. Inguscio, Europhys. Lett. 49, 8 (2000).

[4] D. S. Jin, M. R. Matthews, J. R. Ensher, C. E. Wieman, and E. A. Cornell, Phys. Rev. Lett. 78, 764 (1997).
[5] D. M. Stamper-Kurn, H.-J. Miesner, S. Inouye, M. R. Andrews, and W. Ketterle, Phys. Rev. Lett. 81, 500 (1998).

[6] F. Chevy, V. Bretin, P. Rosenbusch, K. W. Madison, and J. Dalibard, Phys. Rev. Lett. 88, 250402 (2002).

[7] M. Kottke, T. Schulte, L. Cacciapuoti, D. Hellweg, S. Drenkelforth, W. Ertmer, and J. J. Arlt, Phys. Rev. A 72, 053631 (2005).

[8] J. E. Lye, L. Fallani, M. Modugno, D. S. Wiersma, C. Fort, and M. Inguscio, Phys. Rev. Lett. 95, 070401 (2005). 
[9] Y. P. Chen, J. Hitchcock, D. Dries, M. Junker, C. Welford, and R. G. Hulet, Phys. Rev. A 77, 033632 (2008).

[10] C. Fort, F. S. Cataliotti, L. Fallani, F. Ferlaino, P. Maddaloni, and M. Inguscio, Phys. Rev. Lett. 90, 140405 (2003).

[11] H. Moritz, T. Stöferle, M. Köhl, and T. Esslinger, Phys. Rev. Lett. 91, 250402 (2003).

[12] D. S. Hall, M. R. Matthews, J. R. Ensher, C. E. Wieman, and E. A. Cornell, Phys. Rev. Lett. 81, 1539 (1998).

[13] G. Ferrari, M. Inguscio, W. Jastrzebski, G. Modugno, G. Roati, and A. Simoni, Phys. Rev. Lett. 89, 053202 (2002).

[14] G. Modugno, M. Modugno, F. Riboli, G. Roati, and M. Inguscio, Phys. Rev. Lett. 89, 190404 (2002).

[15] F. Ferlaino et al., J. Opt. B: Quantum Semiclass. Opt. 5, S3 (2003).

[16] K. M. Mertes, J. W. Merrill, R. Carretero-Gonzalez, D. J. Frantzeskakis, P. G. Kevrekidis, and D. S. Hall, Phys. Rev. Lett. 99, 190402 (2007).

[17] M. Edwards, P. A. Ruprecht, K. Burnett, R. J. Dodd, and C. W. Clark, Phys. Rev. Lett. 77, 1671 (1996).

[18] S. Stringari, Phys. Rev. Lett. 77, 2360 (1996).

[19] B. Jackson and E. Zaremba, Phys. Rev. Lett. 88, 180402 (2002).

[20] S. A. Morgan, M. Rusch, D. A. W. Hutchinson, and K. Burnett, Phys. Rev. Lett. 91, 250403 (2003).

[21] P. R. Zilsel, Phys. Rev. 79, 309 (1950).

[22] I. N. Adamenko, K. E. Nemchenko, V. A. Slipko, and A. F. G. Wyatt, J. Low Temp. Phys. 34, 279 (2008).

[23] G. Rickayzen, Phys. Rev. 115, 795 (1959).

[24] D. van der Marel, Phys. Rev. B 51, 1147 (1995).

[25] F. Dalfovo, S. Giorgini, L. P. Pitaevskii, and S. Stringari, Rev. Mod. Phys. 71, 463 (1999).

[26] M. R. Matthews, D. S. Hall, D. S. Jin, J. R. Ensher, C. E. Wieman, E. A. Cornell, F. Dalfovo, C. Minniti, and S. Stringari, Phys. Rev. Lett. 81, 243 (1998).

[27] M. Naraschewski and D. M. Stamper-Kurn, Phys. Rev. A 58, 2423 (1998).

[28] R. Meppelink, R. A. Rozendaal, S. B. Koller, J. M. Vogels, and P. van der Straten (submitted to Phys. Rev. A), e-print arXiv:0909.4429v1.

[29] C. Chin, R. Grimm, P. Julienne, and E. Tiesinga, Rev. Mod. Phys. 82, 1225 (2010).
[30] L. Pitaevskii and S. Stringari, Bose-Einstein Condensation (Oxford University Press, Oxford, 2003).

[31] V. M. Pérez-García, H. Michinel, J. I. Cirac, M. Lewenstein, and P. Zoller, Phys. Rev. Lett. 77, 5320 (1996).

[32] The Bogoliubov theory can be used to determine a quantum correction to Eq. (3) which is proportional to $\left(N a^{3} / u_{r}^{2} u_{z}\right)^{1 / 2}$ [44]. For our experimental parameters this term is negligible.

[33] V. M. Pérez-García, H. Michinel, J. I. Cirac, M. Lewenstein, and P. Zoller, Phys. Rev. A 56, 1424 (1997).

[34] P. G. Kevrekidis, G. Theocharis, D. J. Frantzeskakis, and B. A. Malomed, Phys. Rev. Lett. 90, 230401 (2003); S. K. Adhikari, J. Phys. B 36, 1109 (2003); F. K. Abdullaev and J. Garnier, Phys. Rev. A 70, 053604 (2004); F. K. Abdullaev, R. M. Galimzyanov, M. Brtka, and R. A. Kraenkel, J. Phys. B 37, 3535 (2004); E. R. F. Ramos, E. A. L. Henn, J. A. Seman, M. A. Caracanhas, K. M. F. Magalhães, K. Helmerson, V. I. Yukalov, and V. S. Bagnato, Phys. Rev. A 78, 063412 (2008).

[35] S. K. Adhikari, Phys. Lett. A 313, 211 (2003).

[36] S. E. Pollack, D. Dries, M. Junker, Y. P. Chen, T. A. Corcovilos, and R. G. Hulet, Phys. Rev. Lett. 102, 090402 (2009).

[37] S. E. Pollack, D. Dries, and R. G. Hulet, Science 326, 1683 (2009).

[38] C. Menotti and S. Stringari, Phys. Rev. A 66, 043610 (2002).

[39] C. C. Bradley, C. A. Sackett, and R. G. Hulet, Phys. Rev. Lett. 78, 985 (1997).

[40] F. Dalfovo, C. Minniti, and L. P. Pitaevskii, Phys. Rev. A 56, 4855 (1997).

[41] P. Engels, C. Atherton, and M. A. Hoefer, Phys. Rev. Lett. 98, 095301 (2007).

[42] L. P. Pitaevskii, Phys. Lett. A 229, 406 (1997).

[43] S. Giorgini, Phys. Rev. A 61, 063615 (2000).

[44] L. Pitaevskii and S. Stringari, Phys. Rev. Lett. 81, 4541 (1998).

[45] J. Kinast, S. L. Hemmer, M. E. Gehm, A. Turlapov, and J. E. Thomas, Phys. Rev. Lett. 92, 150402 (2004).

[46] A. Altmeyer, S. Riedl, C. Kohstall, M. J. Wright, R. Geursen, M. Bartenstein, C. Chin, J. H. Denschlag, and R. Grimm, Phys. Rev. Lett. 98, 040401 (2007).

[47] S. Riedl, E. R. Sánchez Guajardo, C. Kohstall, A. Altmeyer, M. J. Wright, J. H. Denschlag, R. Grimm, G. M. Bruun, and H. Smith, Phys. Rev. A 78, 053609 (2008). 